From this follows the convergence of (13) for $s<s_{0}$, the interior of the singularity circle, and the convergence of (14) for $s>s_{0}$. The reader may wish to compare this with reference [1], pp. 46-49.

\title{
REFERENCES
}

1. Chaplygin, Gas jets, Moscow 1902, see also NACA TM 1063.

2. Goldstein, Lighthill and Craggs, On the hodrograph transformation for highspeed flow, Q. J. of Mech. Appl. Math. 1, 344-357 (1948).

3. Garrick and Kaplan, On the flow of a compressible fluid by the hodograph method, NACA Report No. 790 (1944).

4. J. Horn, Ueber eine lineare Differentialgleichung zweiter Ordnung mit einem willkuerlichen Parameter, Math. Ann. 52, 271-292 (1899).

\section{ON THE METHOD OF INVERSION IN THE TWO-DIMENSIONAL THEORY OF ELASTICITY*}

\author{
By E. STERNBERG AND R. A. EUBANKS (Illinois Institute of Technology)
}

1. Introduction. The method of inversion, originally introduced by J. H. Michell [1], has led to a variety of technically significant solutions to "plane" problems in the theory of elasticity [2], [3], [4], [5]. The usefulness of Michell's stress-field transformation stems from its invariant properties which assure the preservation of an important class of boundary conditions. In the present note we show that any conformal stress-field transformation which preserves the principal-stress trajectories for every choice of the antecedent Airy function, is essentially a Michell transformation.

2. The Michell transformation. The inversion theorem of Michell may be stated as follows. Let $U(z, \bar{z})$ be real and biharmonic in a region $R$ of the $z$-plane, and let $R^{*}$ be the image of $R$ with respect to the mapping ${ }^{1}$

$$
\zeta=w(z)=\frac{a z+b}{c z+d}, \quad\left|\begin{array}{ll}
a & b \\
c & d
\end{array}\right|=1 .
$$

Then the function

$$
U^{*}(\zeta, \bar{\zeta})=h U[g(\zeta), \overline{g(\zeta)}]
$$

where $h^{2}=\left|w^{\prime}\right|^{2}$ and $g$ is the inverse of $w$, is biharmonic in $R^{*}$. The stress fields generated by $U$ and $U^{*}$, considered as Airy functions in $R$ and $R^{*}$ respectively, are related according to ${ }^{2}$

$$
\left.\begin{array}{c}
\sigma^{*}+i \tau^{*}=\lambda(\sigma+i \tau)+p, \\
\lambda=\frac{1}{h}, \quad p=2\left(\lambda_{z \bar{z}} U-\lambda_{\bar{z}} U_{z}-\lambda_{z} U_{\bar{z}}\right) .
\end{array}\right\}
$$

*Received Mar. 15, 1950.

${ }^{1}$ Michell actually used $\zeta=1 / z$.

${ }^{2}$ Subscripts attached to functions which originally bear no subscripts denote partial differentiation. 
Here $[\sigma, \tau]$ and $\left[\sigma^{*}, \tau^{*}\right]$ are the normal and shearing stresses on any arc $\Gamma$ and on its image $\Gamma^{*}$ with respect to the mapping $\zeta=w(z)$.

Moreover, the stress-field transformation characterized by the mapping (1) and the law of Airy function association (2) has the invariant properties:

(A) The images of the principal stress trajectories of $R$ are the principal stress trajectories of $R^{*}$.

(B) If any arc $\Gamma$ of $R$ is acted on by constant normal tractions only, so is its image $\Gamma^{*}$ of $R^{*}$.

(C) A concentrated load acting at a point $z_{0}$ of a boundary arc $\Gamma$ of $R$, and including a certain angle with $\Gamma$, is carried into a concentrated load of the same magnitude acting at $w\left(z_{0}\right)$ and including the same angle with the image arc $\Gamma^{*}$ of $R^{*}$.

Properties (A) and (B) were also established by V. P. Jensen and D. L. Holl [6] by aid of derivatives of non-analytic (polygenic) functions [7], [8].

3. A converse of the inversion theorem. We now prove the following theorem. Let $R$ be a region of the $z$-plane and let $R^{*}$ be the image of $R$ with respect to the conformal mapping

$$
\zeta=w(z), \quad w^{\prime}(z)=\frac{d w}{d z}=h e^{i \delta} \neq 0 .
$$

Moreover, for every Airy function $U(z, \bar{z})$, bi-harmonic in $R$, let there exist an Airy function $U^{*}(\zeta, \bar{\zeta})$, bi-harmonic in $R^{*}$, such that the corresponding stress-field transformation preserves principal stress trajectories, and the image field of stress is purely hydrostatic only if the antecedent field has the same property. ${ }^{3}$ Then $w(z)$ is given by (1) and $U^{*}$ is given by (2), i.e., the transformation is a Michell transformation.

To establish the theorem, we recall a result of Jensen and Holl [6] who showed that

where

$$
\left.\begin{array}{rl}
\sigma+i \tau & =\gamma_{H}(z, \bar{z}, \theta) \\
& =2 U_{z \bar{z}}+2 U_{\bar{i} \bar{z}} e^{-2 i \theta},
\end{array}\right\}
$$

$$
H_{(z, z)}=2 U_{i}
$$

and $\gamma_{H}$ is the directional derivative of $H$ along $\Gamma, \theta$ being the inclination of $\Gamma$. In view of (5), property (A) is equivalent to the statement

whenever

$$
\gamma_{H^{*}}[w(z), \overline{w(z)}, \theta+\delta]=\bar{\gamma}_{H^{*}}
$$

provided

$$
\gamma_{H}(z, \bar{z}, \theta)=\bar{\gamma}_{H}
$$

$$
H^{*}(\zeta, \bar{\zeta})=2 U_{\bar{\zeta}}^{*}
$$

Equations (7), (8) by aid of (5) become

$$
\left.\begin{array}{l}
U_{i \bar{z}}=U_{z z} e^{4 i \theta} \\
U_{\xi \xi}^{*}=U_{\xi \zeta}^{*} e^{4 i(\theta+\delta)}
\end{array}\right\}
$$

${ }^{3}$ This restriction is essential in order to rule out the trivial transformation which carries all antecedent stress distributions into hydrostatic fields of stress.

"The partial derivatives of $U^{*}$ with respect to $\zeta$ and $\zeta$ are to be evaluated at $\zeta=w(z)$ 
Thus

$$
U_{z z} U_{\xi \xi}^{*}\left(\bar{w}^{\prime}\right)^{2}=U_{\bar{z} \bar{z}} U_{\xi \zeta}^{*}\left(w^{\prime}\right)^{2},
$$

which implies that the function

$$
\phi(z, \bar{z}) \equiv U_{\bar{z} \bar{z}} U_{\zeta \zeta}^{*}\left(w^{\prime}\right)^{2}
$$

is real-valued. Furthermore, assuming that the original stress distribution is not hydrostatic, so that $U_{z z} \neq 0$, it follows by hypothesis that $U_{55}^{*} \neq 0$ and hence $\phi$ does not vanish identically. For convenience, let

$$
\varphi(z, \bar{z})=U_{z z} U_{\bar{z} z} / \phi .
$$

Equation (12) then appears as

$$
U_{z z}=\varphi\left(w^{\prime}\right)^{2} U_{\zeta 5}^{*},
$$

where $\varphi$ is again real-valued. Equation (14) constitutes a necessary and sufficient condition for the preservation of the principal-stress trajectories.

We next apply to (14) the condition that $U(z, \bar{z})$ and $U^{*}(\zeta, \bar{\zeta})$ are both biharmonic, i.e., $U_{z z \bar{z} \bar{i}}=0$ and $U_{\zeta \zeta \xi \xi}^{*}=0$. This leads to

$$
\left[\varphi \frac{w^{\prime \prime}}{w^{\prime}}+2 \varphi_{z}\right] U_{z i \bar{z}}+\left[\varphi_{z z}-\varphi_{z} \frac{w^{\prime \prime}}{w^{\prime}}-\frac{\left(2 \varphi_{z}\right)^{2}}{\varphi}\right] U_{z i}=0,
$$

or, since (15) must hold for every bi-harmonic $U$,

$$
\begin{gathered}
\varphi \frac{w^{\prime \prime}}{w^{\prime}}+2 \varphi_{z}=0, \\
\varphi \varphi_{z z}-\varphi \varphi_{z} \frac{w^{\prime \prime}}{w^{\prime}}-2\left(\varphi_{z}\right)^{2}=0 .
\end{gathered}
$$

The complete solution of (16), subject to the requirement $\varphi=\bar{\varphi}$, is

$$
\varphi(z, \bar{z})=\kappa\left(w^{\prime} \bar{w}^{\prime}\right)^{-1 / 2}=\kappa / h
$$

with $\kappa$ an arbitrary real constant. Noting that (16), (17) require $\varphi_{z z}=0$, we conclude from (18) that

$$
\left(\frac{w^{\prime \prime}}{w^{\prime}}\right)^{\prime}-\frac{1}{2}\left(\frac{w^{\prime \prime}}{w^{\prime}}\right)^{2}=0,
$$

i.e., the Schwarzian derivative of $w(z)$ vanishes. The complete solution of (19) is given by

$$
w(z)=\frac{a z+b}{c z+d}
$$

and since the mapping is to be $(1,1)$ we may put $a d-b c=1$. In order to arrive at the law of Airy-function association we now integrate (14) by use of (18). This integration yields,

$$
U^{*}=\frac{h}{\kappa}[U+A z \bar{z}+\alpha \bar{z}+\bar{\alpha} z+B],
$$


where $A, B$ are arbitrary real constants, and $\alpha$ is an arbitrary complex number. It is readily confirmed by direct computation that the constants $A, B$, and $\alpha$ give rise to an arbitrary, uniform hydrostatic stress-field in the image domain $R^{*}$. The constant $\kappa$, on the other hand, affects merely the scale of the image stress-distribution. We may therefore put $A=B=\alpha=0, \kappa=1$. This completes the proof.

\section{REFERENCES}

1. J. H. Michell, The inversion of plane stress, Proc. London. Math. Soc. 34, 134 (1901).

2. Ludwig Foeppl, Drang und Zwang, vol. 3, Leibniz Verlag, Muenchen, 1947.

3. C. Weber, Spannungsverteilung in Blechen mit mehreren kreisrunden Loechern, ZS. angew. Math. Mech. 2, 267 (1922).

4. W. Olszak, Beitraege zur Anwendung der Inversionsmethode bei Behandlung von ebenen Problemen der Elastizitaetstheorie, Ingenieur Archiv 6, 402 (1935).

5. R. D. Mindlin, Stress systems in a circular disk under radial forces, J. Appl. Mech. 4, 115 (1937).

6. V. P. Jensen and D. L. Holl, An application of derivatives of non-analytic functions in plane stress problems, Bull. Amer. Math. Soc. 43, 256 (1937).

7. Edward Kasner, A new theory of polygenic functions, Science 66, 581 (1927).

8. E. R. Hedrick, Non-analytic functions of a complex variable, Bull. Amer. Math. Soc. 39, 75 (1933).

9. Rufus Isaacs, Planar elasticity as a potential theory, Mathematicas y Fisca Theorica, Universidad Nacional de Tucuman, (A) 6, 263 (1948).

\section{A MINIMUM PRINCIPLE FOR STRUCTURAL STABILITY*}

By H. J. WEISS AND G. H. HANDELMAN (Carnegie Institute of Technology)

1. Statement of the problem. In a recent paper, ${ }^{1} \mathrm{~W}$. Prager has discussed the problem of structural stability from the following point of view. Consider a given configuration of a deformable body, referred to a fixed system of rectangular axes, $x_{i}(i=1,2,3)$, under a set of stresses $\lambda \sigma_{i j}$ which are in equilibrium with given surface tractions. These stresses are prescribed only to within the arbitrary constant factor $\lambda$. The configuration is assumed to be stable if $\lambda$ is sufficiently small. A system of infinitesimal perturbation displacements $u_{i}$ is then applied, and the question is asked for what values of the factor $\lambda$ will the equilibrium become indifferent.

The solution to the problem leads to the following system of linear, homogeneous, second order, partial differential equations

$$
J_{i, i}=0,
$$

subject to the homogeneous boundary conditions on the surface

$$
J_{i j} n_{i}=0,
$$

where

$$
J_{i i}=\left[\tau_{i j}+\frac{1}{2} \lambda\left(\sigma_{i p} \epsilon_{p i}-\sigma_{i p} \epsilon_{p i}\right)-\lambda \sigma_{i p} \omega_{p i}\right]
$$

*Received May 29, 1950.

'W. Prager, The general variational principle of the theory of structural stability, Q. Appl. Math., 4, 378-384 (1947). 